\title{
Influências do Desmame Precoce em Lactentes: Uma Revisão
}

\author{
Influences of Early Weaning in Infants: A Reviewem \\ Influencias del destete temprano en los lactantes: Una revisión
}

Recebido: 27/04/2021 | Revisado: 04/05/2021 | Aceito: 05/05/2021 | Publicado: 20/05/2021

Thayanne Goiana de Souza
ORCID: https://orcid.org/0000-0003-2970-1719
Centro Universitário UNINTA, Brasil
E-mail: nutri.thayanne@gmail.com
Edilayne Gomes Bôto
ORCID: https://orcid.org/0000-0002-8893-5649
Centro Universitário UNINTA, Brasil
E-mail: edylanne88@ hotmail.com
Tamires Alexandre Félix
Coordenação da Atenção Secundária da Secretaria da Saúde de Sobral-CE, Brasil
E-mail: tamiresafelix@ @mail.com
Jorge Luís Rodrigues dos Santos
ORCID: https://orcid.org/0000-0003-1349-6984
Escola de Saúde Pública Visconde de Sabóia, Brasil
E-mail: nutrijorgeluis @ gmail.com
Pedro Ítalo Alves de Carvalho
ORCID: https://orcid.org/0000-0001-5088-1342
Escola de Saúde Pública Visconde de Sabóia, Brasil
E-mail: pedroitaloac@ @mail.com
Jaime Conrado Aragão Neto
ORCID: https://orcid.org/0000-0002-9284-6366
Escola de Saúde Pública Visconde de Sabóia, Brasil
E-mail: Jaimearagaoneto@ hotmail.com
Larisse Araújo de Sousa
ORCID: https://orcid.org/0000-0002-9575-8855
E-mail: larissesousa @ @sobral.ce.gov.br
Francisco Valdicélio Ferreira
ORCID: https://orcid.org/0000-0002-6347-2844
E-mail: celionutri @gmail.com

\begin{abstract}
Resumo
O Aleitamento Materno é de extrema importância nos primeiros seis meses de vida por ser um alimento nutricionalmente completo, não sendo necessária nenhuma complementação. A pesquisa tem como objetivo verificar a influência do desmame precoce em lactentes. Trata-se de uma revisão sistemática de trabalhos publicados nos últimos 10 anos. A busca dos artigos foi realizada em banco de dados eletrônicos como Scientific Eletronic Library Online (Scielo) e Literatura Latino-Americana e do Caribe em Ciências da Saúde (Lilacs). Foram utilizados os descritores "Aleitamento Materno", "Desmame Precoce", "Lactente", "Alergia" e "Doença", totalizando 18 artigos selecionados, no qual foi apontado em todos os estudos que as amostras das pesquisas realizadas obtiveram o inferior a 50\% em relação ao Aleitamento Materno Exclusivo pelo ciclo de seis meses, conforme recomendado pelo Ministério da Saúde. Os fatores mais recorrentes para os desmame precoce foram o uso de chupeta, mamadeira e o trabalho materno fora de casa, ocasionando assim uma possível doença e/ou alergia, pois há evidências para se afirmar que crianças com tempo de aleitamento menor que seis meses, tenham mais propensão para o desenvolvimento dessas alterações em seu estado de saúde. Conclui-se que nos estudos realizados de 2008 a 2018 é possível perceber que o desmame precoce ainda é bastante comum na sociedade. Outro ponto observado na pesquisa foi o predomínio do tempo de aleitamento materno, que foi identificado abaixo da média apontado pela Organização Mundial de Saúde. Diante dessas evidências, recomenda-se o Aleitamento Materno até os dois anos de idade, juntamente com a introdução gradual de uma alimentação complementar a partir do sexto mês de vida.
\end{abstract}

Palavras-chave: Aleitamento materno; Desmame; Lactente; Alergia e imunologia.

\begin{abstract}
Breastfeeding is extremely important in the first six months of life because it is a nutritionally complete food, with no supplementation necessary. The research aims to verify the influence of early weaning on infants. The articles were
\end{abstract}


searched in electronic databases such as ScientificEletronic Library Online (Scielo) and Latin American and Caribbean Literature in Health Sciences (Lilacs). The descriptors "Breastfeeding", "Weaning Precocious", "Infants", "Allergy" and "Disease" were used, totaling 18 articles selected, in which it was pointed out in all studies that the samples of the researches obtained obtained less than $50 \%$ relative to exclusive breastfeeding for the six-month cycle, as recommended by the Ministry of Health. The most recurrent factors for early weaning were the use of pacifiers, bottle feeding and maternal work outside the home, thus causing a possible disease and / or allergy, since there is evidence to affirm that children with breastfeeding less than six months are more likely to develop these changes in their health status. It is concluded that in the studies carried out from 2008 to 2018 it is possible to perceive that early weaning is still quite common in society. Another point observed in the research was the predominance of breastfeeding time, which was identified below the average indicated by the World Health Organization. Given these evidences, breastfeeding is recommended until the age of two, along with the gradual introduction of a complementary feeding from the sixth month of life.

Keywords: Breast feeding; Weaning; Infant; Allergy and immunology.

\section{Resumen}

La lactancia materna es extremadamente importante en los primeros seis meses de vida, ya que es un alimento nutricionalmente completo y no es necesario ningún suplemento. La investigación tiene como objetivo verificar la influencia del destete temprano en los bebés. Se trata de una revisión sistemática de trabajos publicados en los últimos 10 años. La búsqueda de los artículos se realizó en bases de datos electrónicas como Scientific Eletronic Library Online (Scielo) y Literatura Latinoamericana y del Caribe en Ciencias de la Salud (Lilacs). Se utilizaron los descriptores "Lactancia materna", "Destete precoz", "Lactante", "Alergia" y "Enfermedad", totalizando 18 artículos seleccionados, en los que se señaló en todos los estudios que las muestras de investigación obtenidas tenían menos del $50 \%$ en relación con la lactancia materna exclusiva para el ciclo de seis meses, según lo recomendado por el Ministerio de Salud. Los factores más recurrentes para el destete precoz fueron el uso de chupetes, biberones y trabajo materno fuera del hogar, provocando así una posible enfermedad y / o alergia, ya que existe evidencia para afirmar que los niños con tiempo de lactancia menor a seis meses tienen más probabilidades de desarrollar estos cambios en su estado de salud. Se concluye que en los estudios realizados entre 2008 y 2018 es posible notar que el destete precoz sigue siendo bastante común en la sociedad. Otro punto observado en la investigación fue el predominio del tiempo de lactancia, el cual fue identificado por debajo del promedio indicado por la Organización Mundial de la Salud. Ante esta evidencia, se recomienda la lactancia materna hasta los dos años de edad, junto con la introducción paulatina de la alimentación complementaria después del sexto mes de vida.

Palabras clave: Lactancia materna; Destete; Lactante; Alergia e inmunología.

\section{Introdução}

O Leite Materno (LM) é o alimento mais completo e nutritivo que o bebê precisa, sendo de extrema importância nos primeiros seis meses de vida por proporcionar uma melhor qualidade de vida tanto para a criança quanto para a mãe. Sua composição varia de acordo com a idade da criança e durante as mamadas (Barbosa et al., 2009).

Segundo dados da II Pesquisa de Prevalência de Aleitamento Materno (AM) nas Capitais Brasileiras e Distrito Federal, a prevalência de Aleitamento Materno Exclusivo (AME) nos primeiros seis meses de vida foi de $41 \%$. Verificou-se também que houve queda acentuada da probabilidade de crianças com amamentação exclusiva já nos primeiros dias de vida em todas as regiões brasileiras, especialmente na região Nordeste, onde foi encontrada a pior situação, com probabilidade de interrupção do AME em torno de 40\% (Brasil, 2009).

Por outro lado, a amamentação exclusiva até os seis meses de vida é o método que mais previne mortes infantis, além de promover a saúde física, mental e psíquica da criança e da mãe, sendo importante também para a sobrevivência, crescimento, desenvolvimento, saúde e nutrição do bebê (Brasil, 2009).

O Ministério da Saúde ressalta que o LM no segundo ano de vida continua tendo uma fonte de nutrientes. Estima-se que dois copos $(500 \mathrm{~mL})$ de LM durante esse período fornecem $95 \%$ das necessidades de vitamina C, 45\% das de vitamina A, $38 \%$ das de proteína e $31 \%$ do total de energia. Além disso, protege a criança contra doenças infecciosas, tendo menores episódios de diarreia e menor índice de hospitalização por doenças respiratórias (Brasil, 2015).

No entanto, existem alguns fatores que levam as mães a desmamarem seus filhos precocemente, podendo estar relacionado à mãe e/ou a criança. Esse processo de interrupção do AME é definido como desmame precoce e é caracterizado 
pela suspensão do LM por outros alimentos ou leites provenientes de outras fontes (Parizotto \& Zorzi, 2008). O desmame precoce predispõe doenças evitáveis, como desnutrição, diarreia, obesidade infantil, entre outros problemas de saúde pública no mundo, além de favorecer o aumento da mortalidade infantil (Alvarenga et al., 2017).

Salienta-se que a idade materna, o baixo nível de escolaridade, o uso precoce de fórmulas lácteas, chupeta e mamadeira, a falta de incentivo da família e da sociedade, além de deficiências na atenção a saúde são fatores que estão relacionados ao desmame precoce (Ciampo et al., 2006).

Diante ao exposto acerca da realidade brasileira sobre o assunto, justifica-se a elaboração deste estudo com a finalidade de detectar a influência do desmame precoce em crianças menores de seis meses, assim como analisar suas possíveis causas. Portanto, o presente estudo pretende responder aos seguintes questionamentos: Quais possíveis problemas ocorreram durante a lactação que pudessem levar ao desmame precoce? A criança que foi desmamada de forma precoce tem uma maior probabilidade de ter algum tipo de alergia e/ou doença quando comparado àquelas crianças que receberam o Aleitamento Materno Exclusivo?

Com isto este artigo objetiva investigar na literatura científica o tempo de AM e sua influência do desmame precoce assim como suas causas em crianças menores de seis meses de idade e relacionar com a ocorrência de doenças e/ou alergias por meio de uma revisão sistemática.

\section{Metodologia}

Trata-se de uma revisão de literatura, com abordagem exploratória, quantitativa conforme (Brasil, 2012; Gil, 2002; Chizzotti, 2006). Foi utilizado como estratégia de busca para os artigos, as bibliotecas cientificas: Literatura Latino-Americana e do Caribe em Ciências da Saúde (LILACS), Scientific Eletronic Library Online (SCIELO) utilizando as descrições de busca em português como as palavras: Aleitamento Materno, Desmame Precoce, Lactente, Alergia e Doença, e os operadores booleanos “AND” e "OR” conforme demonstrado no Quadro 1 abaixo.

Quadro 1 - Cruzamento dos descritores. Sobral-CE, 2018.

\begin{tabular}{|c|}
\hline Descritores \\
\hline Aleitamento Materno“AND” desmame precoce \\
\hline Aleitamento Mmaterno “AND” Lactente \\
\hline Desmame precoce “AND” Doença “OR” Alergias \\
\hline
\end{tabular}

Fonte: Autores (2018).

Como critérios de inclusão utilizamos pesquisas realizadas no Brasil, que abordassem a influência do desmame precoce em crianças menores de seis meses, publicados nos últimos 10 anos e como critérios de exclusão os artigos com mães que apresentavam alguma patologia que a impossibilitava de amamentar, como HIV (Vírus da Imunodeficiência Humana), mães cujos bebês apresentam paralisia cerebral, lábios leporinos (com fenda no palato e lábios), crianças prematuras, crianças maiores de dois anos ou qualquer problema congênito que tenha impedido o início da amamentação. Para a análise e apresentação dos dados foram selecionados inicialmente os artigos que estavam ligados à temática central, porém, após análise dos critérios de inclusão e exclusão, foram selecionados os estudos. Após a realização do levantamento bibliográfico e seleção das amostras processou-se a leitura dos artigos na íntegra, a fim de proporcionar uma visão global em relação ao assunto.

Os trabalhos selecionados para o presente estudo totalizou em dezoito $(n=18)$ artigos, nos quais foram divididos visando um melhor entendimento das informações contidas nos mesmos. Os dados foram agrupados nas seguintes temáticas: 1. 
"Caracterização geral dos artigos", 2. "Tempo de Aleitamento Materno das amostras", 3. "Causas do desmame precoce de crianças amamentadas com o tempo menor que seis meses", 4. "Relação entre a ocorrência de doenças e/ou alergias e tempo de aleitamento materno".

Os resultados obtidos foram organizados em forma de tabelas no programa Microsoft Word 2010, com intuito de facilitar a analise e alcançar o objetivo proposto.

\section{Resultados e Discussão}

\subsection{Caracterização geral dos artigos}

Através da busca sistemática, foram selecionados 18 artigos de acordo com os descritores, cujo público alvo eram mães com idades entre 12 a 47 anos e crianças com 0 a 6 meses de idade.

Um total de $61,11 \%$ (n=11) artigos abordavam a relação entre o tempo de Aleitamento Materno e 72,22\% ( $n=13$ ) causas do desmame precoce. No entanto, apenas $27,77 \%(n=5)$ artigos abordavam a associação com a ocorrência de doenças e/ou alergias.

Quadro 2 - Bases de dados do presente estudo. Sobral-CE, 2018.

\begin{tabular}{|c|c|c|c|c|}
\hline $\begin{array}{r}\text { Base de } \\
\text { dados }\end{array}$ & Descritores & $\begin{array}{c}\text { Artigos } \\
\text { Encontrados }\end{array}$ & $\begin{array}{l}\text { Pós critérios de } \\
\text { inclusão e exclusão }\end{array}$ & $\begin{array}{c}\text { Artigos } \\
\text { Selecionados }\end{array}$ \\
\hline \multirow{3}{*}{ SCIELO } & $\begin{array}{c}\text { Aleitamento Materno “AND” Desmame } \\
\text { precoce }\end{array}$ & 112 & 47 & 8 \\
\hline & Aleitamento Materno “AND” Lactente & 152 & 99 & 8 \\
\hline & pesmame precoce “AND” Doença e Alergias & 10 & 2 & 1 \\
\hline \multirow[t]{3}{*}{ LILACS } & $\begin{array}{l}\text { Aleitamento Materno “AND” Desmame } \\
\text { precoce }\end{array}$ & 2.170 & 7 & 0 \\
\hline & Aleitamento Materno “AND” Lactente & 25.644 & 9 & 0 \\
\hline & pesmame precoce “AND” Doença e Alergias & 658 & 5 & 1 \\
\hline TOTAL & - & 28.746 & 169 & 18 \\
\hline
\end{tabular}

Fonte: Autores (2018).

\subsection{Tempo de aleitamento materno}

Conforme descrito na Tabela 1, todos os estudos apontaram que menos de 50\% da amostra estudada realizavam o AME pelo período de seis meses, preconizado pelo Ministério da Saúde, dados estes que são considerados como uma situação "ruim" segundo os parâmetros da OMS, (Brasil, 2009). 
Tabela 1 - Tempo de Aleitamento Materno Exclusivo dos Estudos até 180 dias (06 meses).

\begin{tabular}{|c|c|c|}
\hline ESTUDO & IDADE DA MÃE & $\begin{array}{l}\text { PERCENTUAL DAS AMOSTRAS DOS ESTUDOS } \\
\text { CUJAS MÃES AMAMENTARAM ATÉ } 180 \text { DIAS }\end{array}$ \\
\hline FRANCO et al., 2008 & $14-47$ anos & $\mathrm{n}=43,6 \%$ \\
\hline RAMOS et al., 2008 & $12-35$ anos & $\mathrm{n}=16 \%$ \\
\hline CAMPAGNOLO et al., 2012 & Não Citado & $\mathrm{n}=21,4 \%$ \\
\hline LEONE et al., 2012 & Média 26 anos & $\mathrm{n}=39,1 \%$ \\
\hline SALUSTIANO et al., 2012 & $20-35$ anos & $\mathrm{n}=39,7 \%$ \\
\hline SOUZA et al., 2012 & $20-34$ anos & $\mathrm{n}=33,8 \%$ \\
\hline SADECK \& LEONE, 2013 & Não Citado & $\mathrm{n}=39,0 \%$ \\
\hline ROCCI \& FERNANDES, 2014 & $\begin{array}{l}\text { Média } 25,7 \\
\quad \text { anos }\end{array}$ & $\mathrm{n}=34,1 \%$ \\
\hline SALDAN et al., 2015 & $>20$ anos & $\mathrm{n}=36,0 \%$ \\
\hline SCHINCAGLIA et al., 2015 & $20-35$ anos & $\mathrm{n}=4,7 \%$ \\
\hline SANTOS et al., 2016 & $20-34$ anos & $\mathrm{n}=32,0 \%$ \\
\hline
\end{tabular}

Fonte: Autores (2018).

O AM é uma prática de extrema importância para a saúde do bebê, pois proporciona seu crescimento e desenvolvimento de forma saudável. Porém, um estudo realizado com 261 mães na região Sudeste de São Paulo em um Hospital Maternidade Leonor Mendes de Barros, cujo objetivo foi identificar os padrões de AME nos primeiros seis meses de vida, os pesquisadores realizaram a coleta de dados em dois momentos: a primeira ocorreu no ambulatório de retorno do hospital e a segunda foi realizada por meio de entrevistas por telefone durante seis meses. Foi detectado AME de $95 \%$ dos pesquisados até os 30 dias de nascimento, $74 \%$ aos 60 dias, $63 \%$ aos 90 dias, $45 \%$ aos 120 dias, $26 \%$ aos 150 dias e apenas $15 \%$ até os 180 dias (Figueredo et al., 2013).

Por outro lado, numa pesquisa realizada por (Salustiano et al., 2012) envolvendo um estudo com 667 crianças de até seis meses numa Campanha Multivacinação em Uberlândia-MG, foi observado que a prevalência do AM para os bebês entre quatro e seis meses foi de 89,5 \% e 85,0\%, respectivamente. Já na modalidade de AME foi encontrada prevalência de 50,6\% e 39,7\% para menores de 120 e 180 dias respectivamente. Em um contexto mais detalhado, foi averiguado que, das crianças menores de dois meses $95 \%$ receberam AM, tendo uma redução de $10 \%$ entre dois e quatro meses $(84,5 \%)$, e na faixa etária de quatro a seis meses foi encontrado um valor de 77,2\% que ainda recebiam LM.

Neste mesmo sentido, em outra pesquisa realizada com 854 crianças no município de Imperatriz-MA. Foi observado que a prevalência do AME em crianças menores de seis meses foi de 32,0\%. O estudo mostra que a predominância foi menor do que na Capital do Estado, em São Luís- MA com 46,7\% e que a média no Brasil é de 41,0\% (Santos et al., 2016).

Nota-se que, a importância do AM vem sendo extensamente discutida em vários estudos quanto ao grau de proteção e benefícios para o lactente, contudo percebe-se que há uma grande dificuldade em aumentar as taxas de AME. Na cidade de Porto Alegre-RS no ano de 2008 (Campagnolo et al., 2012), realizaram uma pesquisa em postos de vacinação em com 1.099 
crianças menores de um ano de idade, e foi observado que a prevalência de AME no momento da entrevista entre crianças com até quatro meses foi de $47,1 \%$, e crianças de quatro a seis meses foi de $21,4 \%$. Em outro estudo realizado no município de Joinville em Santa Catarina, com 889 Lactentes com idade inferior a um ano de vida, as frequências de AME 53,9\% com em menores de quatro meses e 43,6\% entre quatro a seis meses (Franco et al., 2008). Ao estabelecer um comparativo entre os resultados obtidos nos dois estudos, notou-se uma predominância baixa de AME. Durante a análise dos resultados dos dois estudos relatados percebeu-se que, no decorrer dos anos, o AME está regredindo mesmo com o aumento de ações educativas relacionadas à temática.

Em um estudo transversal realizado (Ramos et al., 2008), em uma Campanha de Vacinação no Piauí, com um total de 1.963 crianças com idade menor ou igual há 364 dias, a probabilidade do AME foi de $16 \%$ ao final de 180 dias e, com relação ao AM, o resultado obtido para a idade de 364 dias foi de 18,6\%. De acordo com as duas probabilidades o autor relata que os valores obtidos foram maiores em relação aos estudos comparados.

Em outro estudo realizado por (Sadeck \& Leone, 2013), foi identificado que a prevalência do AME foi de 52\% até os três primeiros meses e 39\% até os seis meses de vida. Nessa pesquisa foram realizadas 1.225 entrevistas com pais de crianças menores de um ano no município de São Paulo. Observou-se que no primeiro trimestre o AME permaneceu razoavelmente estável, porém após esse período verificou-se uma redução progressiva.

No ano de 2012 em Guarapuava - Paraná, em pesquisa realizada sobre a prevalência do AME em uma campanha nacional de vacinação contra poliomielite com 1.814 crianças também observou-se baixa adesão de $(36,0 \%)$ em crianças menores de seis meses. No mesmo estudo notou-se que crianças amamentadas na primeira hora de vida foi de 79,3\% - taxa definida como um nível bom do município segundo os valores da OMS. No entanto, o autor relata que com exceção do início precoce do AM a situação é considerada ruim ou muito ruim na prática do AME em crianças menores de seis meses (Saldan et al., 2015).

Ao se estabelecer um comparativo entre as pesquisas selecionadas, observou- se que no estudo elaborado na região Noroeste de Goiânia por (Schincaglia et al., 2015) com 362 crianças, foi observado uma diminuição no tempo do AM, onde crianças aos primeiros 30 dias de vida apresentavam uma prevalência de AME de 47,1\%, ja no quarto mês com 22,3\% e no sexto mês de vida com apenas $4,7 \%$.

Uma pesquisa realizada durante o dia da campanha nacional de vacinação com um total de 770 entrevistados (Souza et al., 2012) observaram que a prevalência do AME de crianças de zero a seis meses de idade foi de 33,8\%. Os resultados apresentam maiores nos primeiros meses entre o primeiro e quarto mês $(47,4 \%$ a $62,5 \%)$, porém houve uma queda acentuada do quarto para o quinto mês de 53,7\% para 19,3\% e por essa ordem no sexto mês apenas 7,8\% e relação ao AME.

Da mesma forma, outro estudo realizado em uma campanha nacional de vacinação contra a poliomielite no município de São Paulo, com 704 pais ou responsáveis de crianças, foi observado uma prevalência do AME com 39,1\% em crianças menores de seis meses de idade (Leone et al., 2012).

Em um estudo realizado com 206 mães em um Hospital Amigo da Criança, localizada na Zona Leste de São Paulo, os dados das pesquisas foram coletados em duas etapas. Na primeira as mães foram entrevistadas na alta hospitalar e na segunda etapa, foi realizado por contato telefônico, para a verificação do AM. O monitoramento por telefone teve início 15 dias após a alta hospitalar e prosseguiram até 180 dias após o nascimento do bebê. A prevalência de mães amamentando exclusivamente foi de 34,1\% aos 180 dias (Rocci \& Fernandes, 2014).

Considerando-se a importância do AME e observado nos estudos que a prevalência encontra-se abaixo do que é recomendado pela OMS, torna-se necessária a intensificação das ações de incentivo a essa prática. No entanto, para que isto aconteça, é fundamental além do investimento em ações de capacitações para instruir os profissionais da saúde, para que os mesmos incentivem as mães logo no pré-natal sobre relevância da amamentação exclusiva até os primeiros seis meses de vida. 


\subsection{Causas do desmame precoce}

De acordo com a Tabela 2, observa-se que o trabalho materno fora de casa e o uso de artefatos como mamadeira e chupetas foram os grandes fatores que interferiram para o desmame precoce. Seguindo de introdução precoce de água, chás, sucos, comidas salgadas e por fim os relatos como a falta de leite, leite fraco e problemas mamários. Diante de tantos benefícios contidos no LM, ainda é notório o número de desmame precoce realizado pelas mães.

Tabela 2 - Causas do desmame precoce em crianças menores de 180 dias (06 meses). Sobral-CE, 2018.

\begin{tabular}{|c|c|}
\hline AUTOR & CAUSAS DO DESMAME PRECOCE \\
\hline Santos et al., (2016) & Uso de chupeta e mamadeira. \\
\hline Campos et al., (2015) & Introdução precoce de água e chás. \\
\hline Saldan et al., (2015) & Uso de mamadeiras. \\
\hline Schincaglia et al., (2015) & $\begin{array}{l}\text { Introdução precoce de água, chás, leites, sucos e comidas salgadas; a baixa } \\
\text { escolaridade, ausência do companheiro, gestante-mãe fumante e/ou etilista, a não } \\
\text { realização do pré-natal, o baixo peso ao nascer e os usos de artefatos (chupeta e } \\
\text { mamadeira). }\end{array}$ \\
\hline Martins et al., (2014) & $\begin{array}{l}\text { Introdução precoce de água, chás, sucos, leite em pó, papa salgada e o uso de chupeta } \\
\text { e mamadeira. }\end{array}$ \\
\hline Rocci \& Fernandes (2014) & Leite fraco, pouco leite, trauma mamilar e o trabalho materno fora de casa. \\
\hline Figueredo et al., (2013) & Trabalho materno fora de casa, falta de orientações de pega correta e posição. \\
\hline Campagnolo et al., (2012) & Uso de chupeta e o trabalho materno fora de casa. \\
\hline Leone et al., (2012) & Uso de chupetas e o trabalho materno fora de casa. \\
\hline Salustiano et al., (2012) & O trabalho materno fora de casa e o uso de mamadeira e chupeta. \\
\hline Souza et al., (2012) & Uso de chupeta, mamadeira e o trabalho materno fora de casa. \\
\hline Araújo et al., (2008). & $\begin{array}{l}\text { Falta de leite, leite fraco, problemas mamários, trabalho materno fora de casa e o uso } \\
\text { de chupeta e mamadeira. }\end{array}$ \\
\hline Franco et al., (2008) & Introdução de líquidos e alimentos; o uso de chupeta e o trabalho materno. \\
\hline
\end{tabular}

Fonte: Autores (2018).

Existem várias fatores que dificultam e que impedem as mães de realizar o AM, dentre eles os fatores psicológicos, fisiológicos, culturais e até mesmo a pressão da vida moderna, ocasionando o desmame precoce. No estudo realizado por (Campos et al., 2015), com 309 mães que tiveram filhos no hospital universitário do interior de São Paulo, foi observado à introdução de alguns tipos de líquidos como água e chás, para saciar a sede do bebê e amenizar as dores como cólicas em crianças menores de seis meses. No mesmo estudo foi evidenciado também que as mães mais jovens não tinham o conhecimento mais profundo sobre o LM, ocorrendo mais comumente o desmame precoce.

No estudo de (Figueredo et al., 2013), observou-se que um dos fatores que contribuíram para o desmame precoce foi a intercorrência mamária durante a hospitalização e na consulta de retorno após a alta hospitalar, além do trabalho fora de casa, a falta de orientações de pega e posição, a dificuldade para amamentar e mães sem experiência prévia de amamentação. Já para (Leone et al., 2012), observa-se que os fatores associados à ausência de AME foram o uso de chupeta nas últimas 24 horas e o 
trabalho materno fora de casa.

Em pesquisa realizada por Martins et al., (2014), com objetivo analisar a ingesta de alimentos/líquidos nos seis primeiros meses de vida, observaram que todas as crianças recebiam alimentos, como água, chás, sucos, leite em pó antes dos primeiros quinze dias de vida e papa salgada aos três meses de idade, foi verificado também que a introdução precoce dos alimentos/líquidos teve forte associação ao uso de chupeta e mamadeira (Martins et al., 2014).

Já os autores Rocci E Fernandes (2014), realizaram um monitoramento com mães em AM destacaram que as maiores dificuldades relatadas foram o leite fraco ou pouco leite, trauma mamilar e a volta ao trabalho como a segunda dificuldade mais mencionada pelas mães, ocorrendo assim o desmame precoce. Nota-se que o apoio de uma equipe de saúde é fundamental para o sucesso da amamentação e para prevenção dos traumas mamilares. Em outro estudo realizado por (Salustiano et al., 2012) foi observado que o desmame esteve mais perceptível nos períodos entre quatro a seis meses de vida e os fatores associados foram o trabalho materno fora de casa, além do uso de mamadeira e chupeta, aumentando a prevalência do desmame precoce.

Seguindo a linha de problemas relacionados aos desmame precoce, (Santos et al., 2016) observaram em sua pesquisa que o uso de chupeta e mamadeira foi um fator que influiu negativamente na prática da amamentação, visto que o uso desses contribuíram para o desmame precoce. Em comparação com outros estudos observaram que o uso da chupeta acarreta na diminuição do número de mamadas e, como consequência, a menor estimulação do complexo mamilo-areolar e menor produção de leite, levando assim ao desmame precoce (Saldan et al., 2015).

Em consonância com os outros estudos, (Franco et al., 2008; Campagnolo et al., 2012) verificaram que o uso de chupeta e trabalho materno fora do lar obtiveram uma diminuição no AM. Além disso, os autores salientam que os profissionais de saúde devem reconhecer que a chupeta é um indicador de problemas na amamentação, provocando assim o desmame precoce.

Os autores (Araújo et al., 2008), em pesquisa realizada com onze mães de crianças menores de seis meses, observaram que as maiores dificuldades encontradas para a realização do AM foi a alegação da "falta de leite", "leite fraco", problemas mamários, doenças associadas geralmente a medicamentos utilizados, o trabalho fora de casa e ao oferecimento de chupeta e mamadeira.

Outras situações que influenciam no desmame precoce, conforme citado por (Schincaglia et al., 2015), é a introdução de água, chás e sucos antes do sexto mês de vida, os autores relatam que durante a pesquisa os principais motivos que ocasionaram o desmame precoce foi à baixa escolaridade, ocupação no lar, ausência de companheiro, mãe fumante e/ou etilista, a não realização do pré-natal, baixo peso ao nascer e o uso de artefatos como chupeta e mamadeira.

Neste sentido (Souza et al., 2012) relatam que as predominâncias de AM estiveram associadas à mãe com mais idade e maior escolaridade, que dispõe de licença maternidade com tempo adequado e possuem mais de um filho. Já a introdução de bicos artificiais (mamadeira, chupeta) e a volta ao trabalho esteve associada à menor chance das crianças serem amamentadas exclusivamente. Contudo o uso da chupeta está relacionado à menor frequência de mamada e, consequentemente, a diminuição do LM, podendo assim ocasionar o desmame precoce.

Nota-se que muitos são os fatores que contribuem para o desmame precoce, o que é um ponto muito preocupante. Destaca-se o uso de artefatos, além do trabalho materno fora de casa. No entanto, deve-se fortalecer as orientações em relação à ordenha e armazenamento do LM antes da volta ao trabalho, sendo um método que contribuirá para a realização do AME até o sexto mês de vida.

Importante relatar ainda que o Ministério da Saúde vem investindo e unindo forças para a melhoria destes dados referente ao AME com adoção de estratégias na atenção primária a saúde como a publicação do manual de implementação da Estratégia Nacional para Promoção do AM e Alimentação Complementar Saudável no Sistema Único de Saúde em busta do 
estímulo a adoção do AME através da Estratégia Amamenta e Alimenta Brasil (Brasil, 2015).

\subsection{Ocorrências de doenças e/ou alergias com o tempo de aleitamento materno.}

Na Tabela 3 pode-se observar a ocorrência de doenças e/ou alergias em crianças menores de seis meses. No entanto as crianças que foram desmamadas precocemente apresentaram problemas de saúde como: aumento de diarreia, desnutrição e distúrbios respiratórios. Em um único estudo foi constatado que crianças com diagnóstico de pneumonia ou bronquiolite com introdução de fórmulas láctea não tiveram associação com o quadro clínico da criança.

Tabela 3 - Ocorrências de doenças e/ou alergias em crianças menores de 180 dias (6 meses). Sobral-CE, 2018.

\begin{tabular}{l|l}
\hline \multicolumn{1}{c|}{ AUTOR } & \multicolumn{1}{c}{ RESULTADOS } \\
\hline Santos et al., (2016) & $\begin{array}{l}\text { Crianças menores de seis meses amamentadas exclusivamente tiveram menos chances de } \\
\text { apresentar episódios de diarreia aguda e as não amamentadas apresentaram 2,6 vezes mais } \\
\text { chances de diarreia. }\end{array}$ \\
\hline Schincaglia et al., (2015) & $\begin{array}{l}\text { Foi observado que a maioria das mães eram fumantes, ocasionando a diminuição da secreção } \\
\text { láctea em crianças menores de seis meses e com um aumento da incidência de doenças } \\
\text { respiratórias. }\end{array}$ \\
\hline Boccolini et al., (2012) & $\begin{array}{l}\text { O percentual de internações por diarreia diminuiu pela metade, representando 2,67\% do total de } \\
\text { internações em 2008. O autor relata que as diarreias podem ser responsáveis por 35\% a 86\% das } \\
\text { mortes infantis evitáveis, sendo que o AMpode ser responsável pela redução de 9,3\% do } \\
\text { coeficiente de mortalidade infantil. }\end{array}$ \\
\hline Araújo et al., (2008) & $\begin{array}{l}\text { Houve aumento de crianças menores de seis meses que foram desmamadas precocemente com } \\
\text { infecção intestinal, desnutrição e distúrbios respiratórios. }\end{array}$ \\
\hline Souza et al., (2008). & $\begin{array}{l}\text { Crianças de até 120 dias (4 meses) com diagnostico de pneumonia ou bronquiolite, foi } \\
\text { observado que o leite materno não se associou com a duração de internação, mesmo as crianças } \\
\text { que permaneceram pouco tempo hospitalizadas e que a introdução de formulas lácteas não se } \\
\text { relacionou com a gravidade do quadro clinico da criança. }\end{array}$ \\
\hline
\end{tabular}

Fonte: Autores (2018).

A ocorrência de doenças e/ou alergias com o tempo de AM foi detectado em apenas cinco dos estudos inclusos na amostra.

De acordo com (Santos et al., 2016), em uma pesquisa realizada no município de Imperatriz - MA com crianças menores de doze meses, foi aplicado um questionário com as mães e, em seguida, realizado o teste qui-quadrado para associar os tipos de AM e o número de episódios de diarreia aguda. Foi observado que as crianças menores de seis meses amamentadas exclusivamente tiveram menos chances de apresentar episódios de diarreia aguda e as não amamentadas apresentaram 2,6 vezes mais chances de diarreia.

Esses resultados são compatíveis com os achados por (Boccolini et al., 2012) em seu estudo ecológico em 18 capitais brasileiras e Distrito Federal entre os anos de 1999 e 2008, com crianças menores de um ano, no qual foi averiguado que com relação ao total de internações hospitalares registradas em 1999, o percentual de internações por diarreia diminuiu pela metade, representando $2,67 \%$ do total de internações em 2008. O autor relata que as diarreias podem ser responsáveis por 35\% a $86 \%$ das mortes infantis evitáveis, sendo que o AM pode ser responsável pela redução de 9,3\% do coeficiente de mortalidade infantil.

Já no estudo de (Schincaglia et al., 2015), foi evidenciado que a introdução de alimentos antes dos seis meses de vida, 
como o leite de vaca poderia desencadear processos alérgicos, além disso, a exposição prematura a proteína diferente do leite humano está associada ao aumento do risco de diabetes tipo 1 e de doenças "atópicas", como asma. No entanto, o autor afirma que a maioria das mães eram fumantes, ocasionando a diminuição da secreção láctea e aumento da incidência de doenças respiratórias.

Há ainda outro estudo, realizado no hospital pediátrico em Salvador - BA no qual foi aplicado um questionário com as mães ou responsáveis pelas crianças ( $\mathrm{n}=97$ ) com até quatro meses de idade, com diagnóstico de pneumonia ou bronquiolite. Observou-se na pesquisa que a interrupção do AME não se associou com a duração da internação, mesmo as crianças que permaneceram pouco tempo hospitalizadas. Além disso, os resultados do estudo revelaram que a introdução de fórmulas lácteas não se relacionou com a gravidade do quadro clinico da criança (Souza et al., 2008).

E por fim, no estudo realizado por (Araújo et al., 2008) no qual realizaram um questionário com mães de crianças menores de seis meses, e observaram que houve aumento de crianças doentes com infecção intestinal, desnutrição e distúrbios respiratórios que foram desmamadas precocemente. Verificaram ainda que as mães tem o conhecimento sobre os valores imunológicos do LM para evitar doenças, no entanto as lactantes se deparam com tantas dificuldades, que a impossibilitam de realizar o AME (Araújo et al., 2008).

\section{Considerações Finais}

O estudo possibilitou investigar a influência do desmame precoce em crianças menores de seis meses, a partir do levantamento bibliográfico LILACS e SCIELO, no qual foi possível identificar o tempo de AM nas pesquisas, os fatores predominantes para o desmame precoce e a semelhança de doenças/alergias em crianças menores de seis meses.

Foi possível observar que o AME está presente em todos os artigos por ser indispensável para a saúde dos Lactentes. No entanto, nota- se que prevalência do tempo de AM nos estudos selecionados encontra-se abaixo do que a Organização Mundial de Saúde preconiza.

Acrescenta-se ainda que ao longo dos estudos, o desmame precoce encontra-se presente e que é causado por múltiplos fatores os quais possuem grande influência na duração do AME. Embora existam várias estratégicas motivadoras para a realização do AME, infelizmente essa prática ainda é um desafio no que diz respeito à recomendação.

Sobre os estudos que abordaram a ocorrência de doenças e/ou alergias em crianças menores de seis meses em desmame precoce foi observado que as mesmas estiveram mais propícias as doenças/alergias, pois na composição do LM existem fatores imunológicos para a proteção dos Lactente. No entanto, apenas um estudo observou que a suspensão do AME em crianças hospitalizadas não demonstrou nenhuma associação com ocorrência de doenças e/ou alergias.

Por fim, verifica-se que os objetivos do estudo foram alcançados, uma vez que foi possível identificar as causas do desmame precoce juntamente com a ocorrência de doenças e/ ou alergias em crianças menores de seis meses.

\section{Referências}

Araújo, O. D., Cunha, A. L., Lustosa, L. R., Nery, I. S., Mendonça, R. C. M. \& Campelo, S. M. A. (2008). Aleitamento materno: fatores que levam ao desmame precoce. Rev. Bras. Enfermagem, 61 (4), 488-92.

Alvarenga, S. C., Castro, D. S., Leite, F. M. C., Brandão, M. A. G., Zandonade, E. \& Primo, C. C. (2017). Fatores que influenciam o desmame precoce. Aquichan, 17(1), 93-103.

Barbosa, M. B., Palmas, D., Domene, S. M. A., Taddei, J. A. C. \&Lopes, F. A. (2009). Fatores de riscos associados ao desmame precoce e ao período de desmame em lactente matriculados em creches. Rev. Paul. Pediatria, 27 (3), 272-281. 2009.

Boccolini, C. S., Boccolini, P. M. M., Carvalho, M. L. \& Oliveira, M. I. C. (2012). Padrões de Aleitamento Materno Exclusivo e internação por diarreia entre 1999 e 2008 em capitais brasileiras. Ciência \& Saúde Coletiva, 17 (7), 1857-1863.

Brasil, Ministério da Saúde. Saúde da Criança: Nutrição Infantil - AMe Aleitamento Complementar. Departamento de Atenção Básica. Caderno de Atenção Básica. http://bvsms.saude.gov.br/bvs/publicacoes/saude_crianca_nutricao_aleitamento_ali mentacao.pdf. 
Brasil. Ministério da Saúde. Secretaria de Atenção à Saúde. Estratégia Nacional para Promoção do AMe Alimentação Complementar Saudável no Sistema Único de Saúde: manual de implementação / Ministério da Saúde, Secretaria de Atenção à Saúde. Ministério da Saúde, 2015. http://bvsms.saude.gov.br/bvs/publicacoes/estrategia_nacional_promocao_aleitamento_materno.pdf

Brasil. Ministério da Saúde. II Pesquisa de prevalência de AMnas capitais brasileiras e Distrito Federal. Brasília: Ministério da Saúde, 2009. http://bvsms.saude.gov.br/bvs/publicacoes/pesquisa_prevalencia_aleitamento_mat erno.pdf .

Brasil. Ministério da Saúde. Conselho Nacional de Saúde. Resolução $\mathrm{N}^{\circ}$ 466, de 12 de dezembro de 2012. https://bvsms.saude.gov.br/bvs/saudelegis/cns/2013/res0466_12_12_2012.html

Brasil. Ministério da Saúde. Secretaria de Atenção à Saúde. Departamento de Atenção Básica. Saúde da criança : AMe alimentação complementar / Ministério da Saúde, Secretaria de Atenção à Saúde, Departamento de Atenção Básica. (2a ed.), Ministério da Saúde, 2015. http://bvsms.saude.gov.br/bvs/publicacoes/saude_crianca_aleitamento_materno_ca b23.pdf.

Campagnolo, P. D. B., Louzada, M. L. C., Silveira, E. L. \& Vitolo, M. R. (2012) Práticas alimentares no primeiro ano de vida e fatores associados em amostra representativa da cidade de Porto Alegre, Rio Grande do Sul. Rev. Nutr., Campinas, 25 (4), 431-439.

Campos, A. M. S., Chaoul, C. O., Carmona, E. V., Higa, R. \& Vale, I. N. (2015). Prática de Aleitamento Materno Exclusivo informado pela mãe e oferta de líquidos aos seus filhos. Rev. Latino-Am. Enfermagem, 23 (2), 283-90.

Chizzotti, A. (2006). A pesquisa qualitativa e seus pensamentos filosóficos. Pesquisa qualitativa em ciências humanas e sociais. Petrópolis: Vozes.

Ciampo, L. A. D., Junqueira, M. J. G., Ricco, R. G., Daneluzzi, J. C., Ferraz, I. S. \& Junior, C. E. M. (2006). Tendência secular do Aleitamento Materno em uma unidade de atenção primaria à saúde materno-infantil em Ribeirão Preto, São Paulo. Revista Brasileira de Saúde Materno Infantil, 6 (4), $391-6$.

Figueredo, S. F., Mattar, M. J. G. \& Abrão A. C. F. V (2013). Hospital Amigo da Criança: prevalência de Aleitamento Materno Exclusivo aos seis meses e fatores intervenientes. Rev. Esc. Enfer, 47 (6), 1291-7.

Franco, S. C., Nascimento, M. B. R., Reis, M. A. M., Issler, H. \& Grisi, S. J. F. E. (2008). Aleitamento Materno Exclusivo em Lactente atendidos na rede pública do município de Joinville, Santa Catarina, Brasil. Rev. Bras. Saúde Materno Infantil, 8 (3), 291-297.

Gil, A. C. (2002). Como elaborar projetos de pesquisa. (4a ed.), Atlas.

Leone, C. R., Sadeck, L. S. R. \& Paulistana, P. R. P. M. (2012). Fatores de risco associados ao desmame em crianças até seis meses de idade no município de São Paulo. Rev. Paul. Pediatria, 30 (1), 21-26.

Martins, C. B. G., Santos, D. S., Lima, F. C. A. \& Gaiva, M. A. M. (2014). Introdução de alimentos para Lactente considerados de risco ao nascimento. Epidemiol. Serv. Saúde, 23 (1), 79-90.

Parizotto, J. \& Zorzi, N. T. (2008). Aleitamento Materno: fatores que levam ao desmame precoce no município de Passo Fundo,RS. Mundo saúde (Impr.), 32 (4), 466-474.

Ramos, C. V., Almeida, J. A. G., Alberto, N. S. M. C., Teles, J. B. M. \& Saldiva, S. R. D. M. (2008). Diagnóstico da situação do Aleitamento Materno no Estado do Piauí, Brasil. Cad. Saúde Pública, 24 (8), 1753-1762.

Rocci, E. \& Fernandes, R. A. Q. (2014). Dificuldades no Aleitamento Materno e influência no desmame precoce. Rev. Bras. Enferm, 67 (1), $22-27$.

Sadeck, L. S. R. \& Leone, C. R. (2013). Avaliação da situação do Aleitamento Materno em menores de um ano de idade no Município de São Paulo, Brasil, em 2008. Cad. Saúde Pública, 29 (2), 397-402.

Santos, F. S., Santos, L. H., Saldan, P. C., Santos, F. C. S., Leite, A. M. \& Mello, D. F. (2016). Aleitamento Materno e diarreia aguda entre crianças cadastradas na estratégia saúde da família. Texto Contexto Enfermagem, 25 (1), 02-08.

Saldan, P. C., Venancio, S. I., Saldiva, S. R. D. M., Pina, J. C. \& Mello, D. F. (2015). Práticas de Aleitamento Materno de crianças menores de dois anos de idade com base em indicadores da Organização Mundial da Saúde. Rev. Nutr., 28 (4), 409-420.

Salustiano, L. P. Q., Diniz, A. L. D., Abdallah, V. O. S. \& Pinto, R. M. C. (2012). Fatores associados à duração do Aleitamento Materno em crianças menores de seis meses. Rev. Bras. Ginecol. Obstetrícia, 34 (1), 28-33.

Schincaglia, R. M., Oliveira, A. C., Sousa, L. M. \& Martins, K. A. (2015). Práticas alimentares e fatores associados à introdução precoce da alimentação complementar entre crianças menores de seis meses na região noroeste de Goiânia. Epidemiol. Serv. Saúde, Brasília, 24 (3), 465-474.

Souza, E. L., Silva, L. R., Sá, A. C. S., Bastos, C. M., Diniz, A. B. \& Mendes, C. M. C. (2008). Impacto da internação na prática do AMem hospital pediátrico de Salvador, Bahia, Brasil. Cad. Saúde Pública, 24 (5), 1062-1070.

Souza, S. N. D. H., Migoto, M. T., Rossetto, E. G. \& Mello, D. F. (2012). Prevalência de AMe fatores associados no município de Londrina-PR*. Acta Paul Enferm, 25 (1), 29-35. 